'Programa Enfermedad Inflamatoria Intestinal. Clínica Las Condes. Santiago, Chile. 2Departamento de Gastroenterología. Clínica Las Condes. Santiago, Chile. ${ }^{3}$ Subdirección Académica. Clínica Las Condes. Santiago, Chile. ${ }^{4}$ Unidad de Coloproctología, Departamento de Cirugía. Clínica Las Condes. Santiago, Chile. ${ }^{5}$ Servicio de Anatomía Patológica. Clínica Las Condes. Santiago, Chile.

${ }^{6}$ Servicio de Imagenología. Clínica Las Condes. Santiago, Chile. ${ }^{7}$ Servicio de Hematología, Hospital Clínico Pontificia Universidad Católica de Chile. Santiago, Chile. aEnfermera.

Conflicto de intereses: No existen conflictos de intereses.

Recibido el 11 de agosto de 2017 aceptado el 14 de noviembre de 2017.

Correspondencia a: Rodrigo Quera Clínica Las Condes, Estoril 450, Las Condes, Santiago, Chile. rquera@clc.cl

\section{Linfoma difuso de células grandes B rectal en un paciente con colitis ulcerosa}

\author{
RODRIGO QUERA ${ }^{1,2}$, LILIAN FLORES ${ }^{1,2, a}$, DANIELA SIMIAN ${ }^{1,3, a}$, \\ UDO KRONBERG ${ }^{1,4}$, MARÍA TERESA VIAL ${ }^{5}$,
}

DAVID LADRÓN DE GUEVARA ${ }^{6}$, MARÍA JOSÉ GARCÍA-RODRÍGUEZ ${ }^{7}$

\section{Rectal diffuse large B cell lymphoma appearing after immunosuppression for ulcerative colitis. Report of one case}

Primary colorectal lymphoma is a rare form of presentation of gastrointestinal tract lymphomas. Inflammatory bowel disease and its treatment are risk factors for its development. We report a 47-year-old male patient with Ulcerative Colitis of two years of evolution, treated initially with azathioprine and later on with infliximab. Due to a relapse in symptoms after the second dose of infliximab, a new coloncoscopy was performed showing a rectal ulcerative lesion, corresponding to a large cell Non-Hodgkin's Lymphoma. The patient was successfully treated with RCHOP chemotherapy (Rituximab cyclophosphamide doxorubicin vincristine prednisone). He is currently in disease remission.

(Rev Med Chile 2017; 145: 1342-1348)

Key words: Colitis, Ulcerative; Crohn Disease; Inflammatory Bowel Disease; Lymphoma, Large B-Cell, Diffuse.
E 1 linfoma del tracto gastrointestinal primario (LGIP) describe al linfoma que se origina en el tracto gastrointestinal, representando sólo $5-10 \%$ de todos los linfomas ${ }^{1,2}$ y hasta $20 \%$ de los linfomas extranodales ${ }^{3,4}$. Este tipo de linfoma, sin embargo, es infrecuente, constituyendo solo 1-4\% de las neoplasias gastrointestinales ${ }^{5}$. Estudios han planteado que la incidencia del LGIP ha aumentado ${ }^{3,6}$, tendencia que podría ser explicada por la presencia de estados de inmunosupresión, ya sea mediada por un síndrome de inmunodeficiencia adquirida (SIDA), por fármacos en contexto de un postrasplante $\mathrm{o}$ asociados a enfermedades autoinmunes como la artritis reumatoidea, lupus eritematoso sistémico o enfermedad celíaca ${ }^{3,7,8}$. Entre los fármacos que favorecen el desarrollo del linfoma destacan los tiopurínicos (azatioprina y 6-mercaptopurina $)^{9,10} \mathrm{y}$, en menor medida, la terapia biológica anti-TNF ${ }^{11}$.
Aunque el LGIP puede comprometer cualquier región del tracto gastrointestinal, las áreas más afectadas son el estómago, seguido del intestino delgado y la región ileocecal ${ }^{12}$. Aunque la región colorrectal puede verse comprometida hasta en $20 \%$ de los LGIP ${ }^{4,12,13}$, el linfoma primario colorrectal es muy poco frecuente, constituyendo $<1 \%$ de las neoplasias a este nivel ${ }^{13}$. Similar a lo que ocurre en otras áreas del tracto gastrointestinal, el linfoma primario colorrectal es principalmente un linfoma no-Hodgkin de células B, siendo el linfoma difuso de células grandes $\mathrm{B}$ el más frecuente. También se puede presentar como un linfoma folicular, linfoma asociado a tejido linfoide de la mucosa, linfoma de células del manto y linfocítico de células pequeñas ${ }^{14,15}$.

Nuestro objetivo es describir, a través de un caso clínico, las características clínico-patológicas y manejo del linfoma colorrectal primario en pacientes con EII. 


\section{Caso clínico}

Hombre de 47 años con antecedente de colitis ulcerosa (CU) extensa diagnosticada en 2014, sin respuesta a mesalazina en dosis de 4,5 g/día, motivo por el cual se agrega azatioprina en dosis de $150 \mathrm{mg} /$ día en noviembre de 2015. Pese a tratamiento inmunosupresor adecuado, el paciente persiste con síntomas digestivos, destacando deposiciones líquidas con sangre y coágulos intermitentes, en una frecuencia de hasta tres episodios diarios (índice de Mayo clínico 5). Se realiza colonoscopía en julio de 2016 que muestra compromiso inflamatorio de recto y sigmoides con un índice de Mayo endoscópico 3 y una enterografía por resonancia de abdomen-pelvis que muestra engrosamiento parietal difuso de aspecto inflamatorio de rectosigmoides (Figura $1 \mathrm{a}-\mathrm{b})$. Por lo referido, se inicia tratamiento con infliximab $5 \mathrm{mg} / \mathrm{kg}$ asociado a azatioprina en septiembre de 2016, evolucionando con una remisión clínica tras la primera dosis (índice de Mayo clínico 1). Dos semanas después de la 2a dosis de infliximab, el paciente refiere dolor en la región rectal asociado a sensación de pujo y de- posiciones líquidas con sangre y coágulos, motivo por el cual se realiza una colonoscopía (octubre de 2016) que muestra una gran lesión ulcerada de bordes elevados y áreas con necrosis (Figura 2 a-c). Las biopsias muestran proliferación linfoide atípica con área de necrosis e inmunomarcación CD20(+), CD30(+), BCL2(+) leve focal, Ki67 $80 \%$, CD3(-), CD10(-), c-MYC(-) y BCL6(-) (Figura 3). Las células tumorales fueron positivas para el virus de Epstein Barr (VEB) determinado a través de hibridación in situ. En el estudio de médula ósea no se observaron elementos de clonalidad sugerentes de compromiso medular por un síndrome linfoproliferativo. La resonancia nuclear magnética de abdomen-pelvis mostró un engrosamiento parietal masiforme del recto medio compatible con un proceso neoplásico que infiltra la grasa mesorectal (Figura $1 \mathrm{c}-\mathrm{d}$ ). El estudio por PET/CT demostró engrosamiento hipermetabólico parietal excéntrico de recto medio de aspecto neoplásico (Figura $4 \mathrm{a}$-c), sin adenopatías ni otros focos hipercaptantes extracolónicos. La serología para VIH, VHB y VHC fue negativa. Con el estudio referido se planteó el diagnóstico de linfoma no-Hodgkin

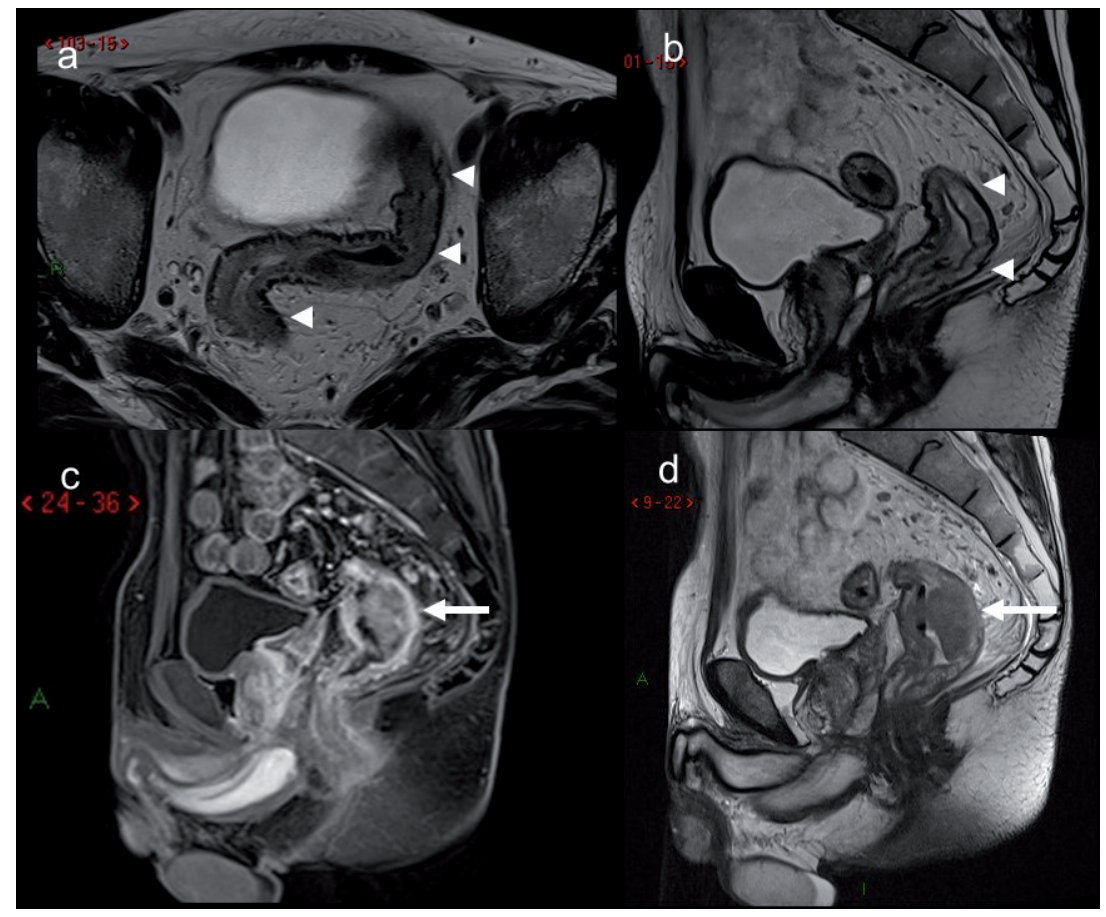

Figura 1. Secuencias $T 2$ de resonancia magnética $\mathbf{( a , b )}$ muestran engrosamiento parietal difuso de aspecto inflamatorio del rectosigmoides, sin masas evidentes (cabezas de flechas). Secuencias T1 gadolinio (c) y T2 (d) tomadas siete semanas después, con engrosamiento parietal masiforme del recto (flechas). 

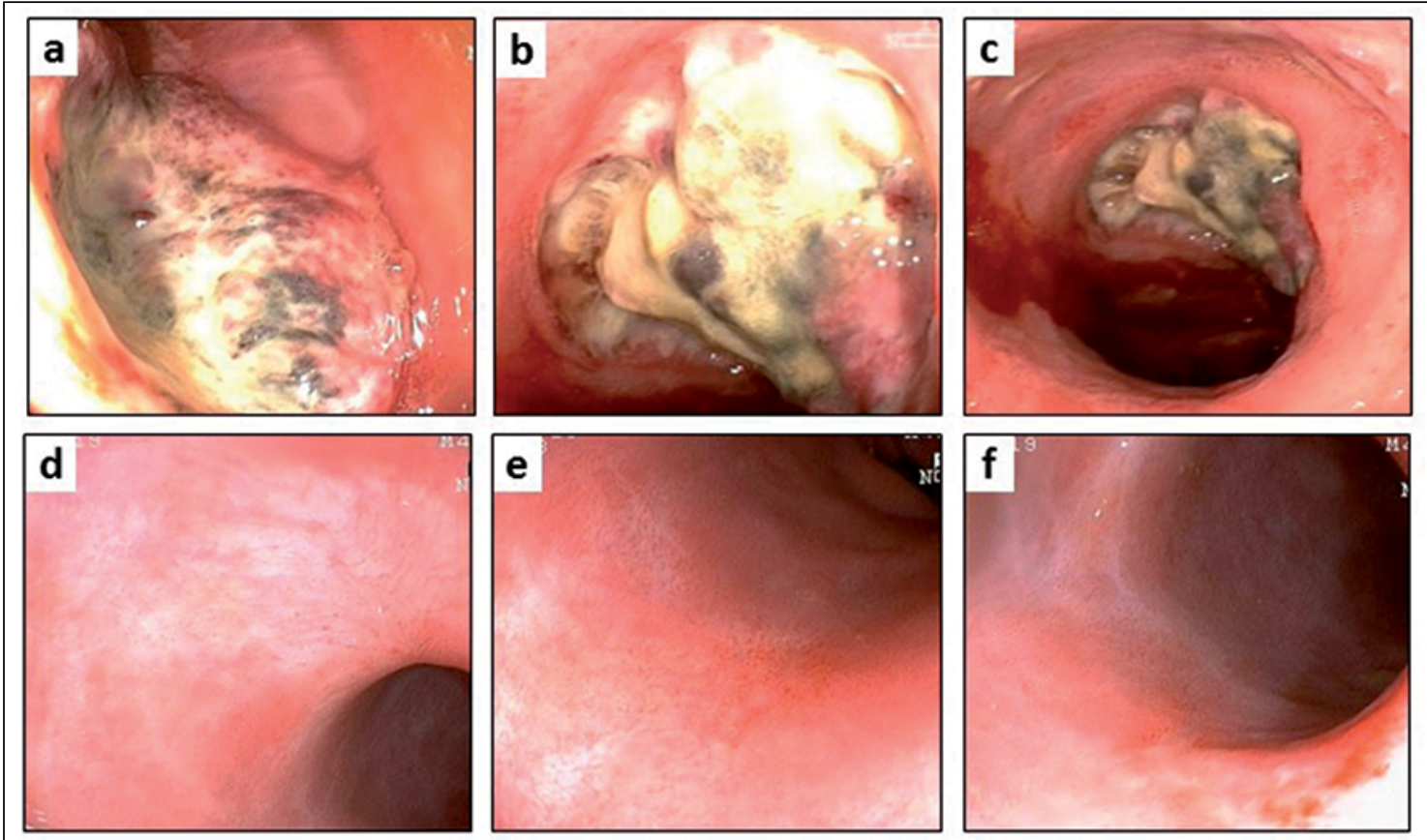

Figura 2. Linfoma no-Hodgkin difuso de células grandes $B$ de recto en paciente con antecedentes de colitis ulcerosa en examen colonoscópico. a-c: lesión previa a quimioterapia y d-f: mucosa rectal post quimioterapia.

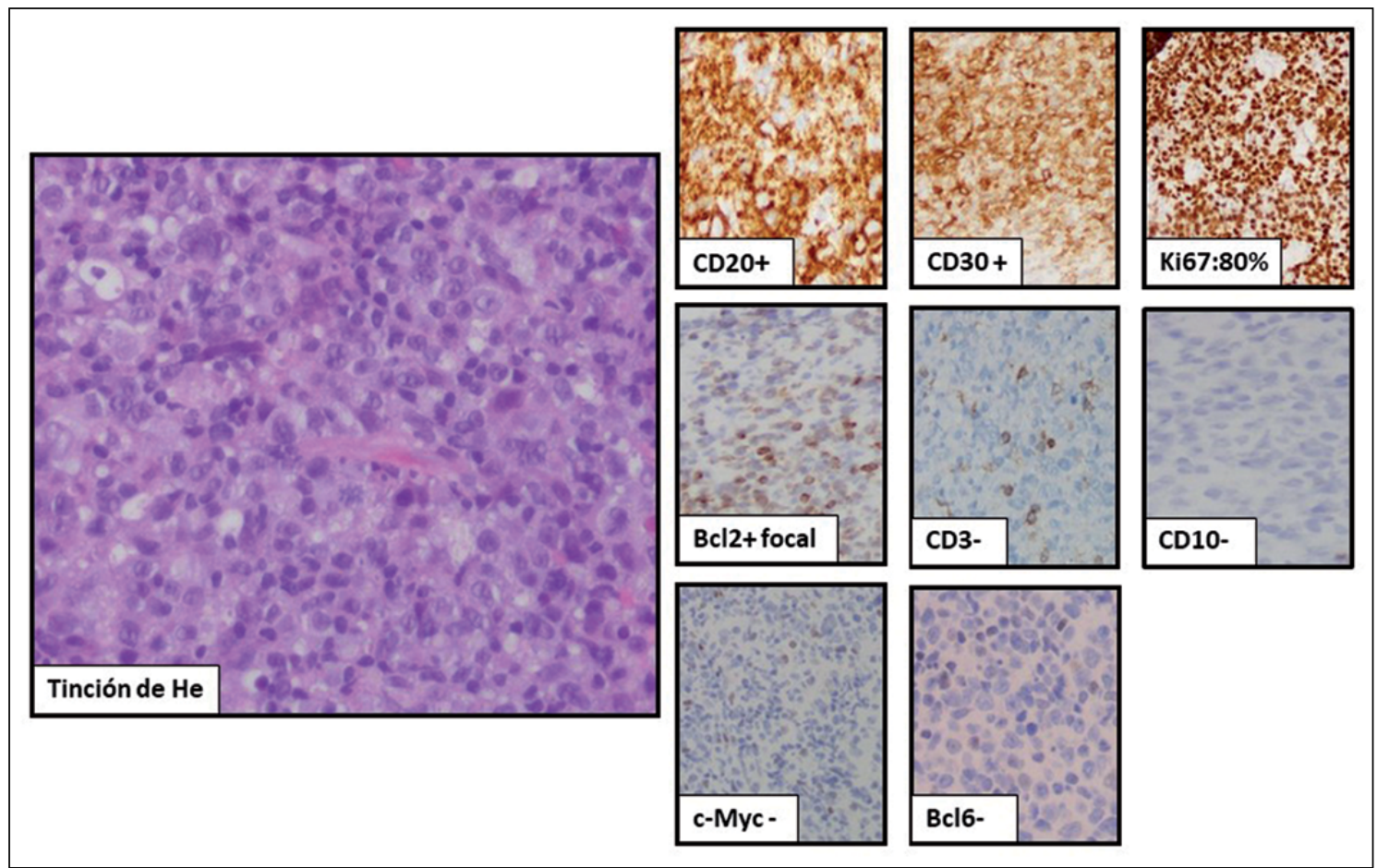

Figura 3. Hallazgos histológicos e inmunomarcación de linfoma no-Hodgkin difuso de células grandes B. A la izquierda, tumor conformado por células linfoides grandes atípicas con núcleos pleomórficos y nucléolo prominente. A la derecha, técnicas de inmunohistoquímica. 


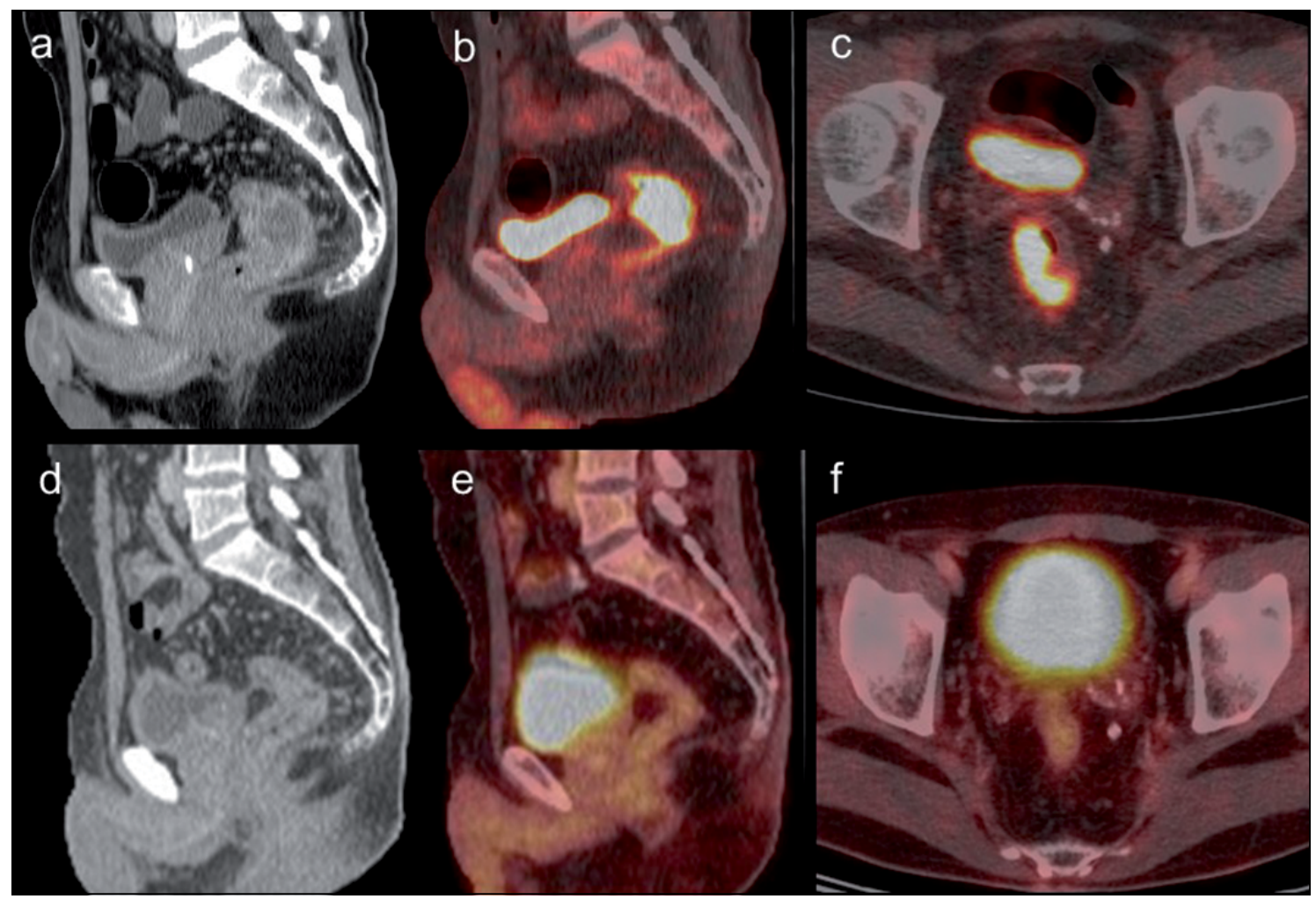

Figura 4. Tomografía computada $(\mathbf{a})$, y $\operatorname{PET} / C T(\mathbf{b}, \mathbf{c})$ inicial, donde se muestra engrosamiento parietal excéntrico del recto (flechas), ávido de F18-FDG. TC y PET/CT post tratamiento sin lesiones metabólicamente activas en recto (d,e,f).

difuso de células grandes B anaplásico etapa I-E con un índice pronóstico internacional revisado (R-IPI) de 0. En este contexto, se suspende azatioprina e infliximab, se indica mesalazina $4,5 \mathrm{~g} /$ día e inicia quimioterapia en diciembre de 2016 con R-CHOP [ciclofosfamida, doxorrubicina, vincristina y prednisona asociado al anticuerpo monoclonal (rituximab)]. Después de 6 ciclos de quimioterapia sin complicaciones, el paciente evoluciona en forma favorable con regresión de sus síntomas generales y digestivos (índice de Mayo clínico 0). Se realiza control con PETCT (junio de 2017) que muestra una respuesta completa, sin masas ni lesiones hipercaptantes en recto (Figura $4 \mathrm{~d}$-f). La colonoscopía realizada en junio de 2017 demostró una regresión completa de la lesión tumoral y un índice de Mayo endoscópico 1 a nivel de recto y sigmoides (Figura 2 d-f). Las biopsias de recto sólo mostraron un compromiso inflamatorio crónico con focos de actividad aguda focal leve, sin evidencias de neoplasia. Se decide mantener tratamiento con mesalazina $4,5 \mathrm{~g} /$ día vía oral asociado a supositorio de mesalazina $500 \mathrm{mg}$ noche, manteniéndose en remisión clínica hasta la fecha.

\section{Discusión}

El linfoma rectal primario es infrecuente, constituyendo solo $0,05 \%$ de todas las neoplasias primarias de recto ${ }^{16}$. Su frecuencia es menor que el linfoma primario de colon ascendente y ciego, dada la mayor cantidad de tejido linfoide en esa área en comparación al resto del colon y recto ${ }^{7}$. La presentación clínica, imagenológica y endoscópica son inespecíficas, por lo que el diagnóstico es difícil, siendo la mayoría de las veces indistinguibles de otras enfermedades del colon, ya sea neoplásicas o inflamatorias. Cuando el linfoma se encuentra en el recto, las principales manifestaciones son sangrado, dolor rectal, sensación de pujo y cambio 
en el hábito intestinal (diarrea o constipación) ${ }^{17}$. En algunos casos, el diagnóstico es realizado en forma incidental, tal como se presentó en nuestro paciente ${ }^{18}$. Aunque la mayoría de los linfomas colorrectal primarios son linfomas no-Hodgkin de células B, estudios realizados en China y Corea han demostrado un mayor porcentaje de linfoma de células $\mathrm{T}$, los cuales tendrían un peor pronósti$\mathrm{Co}^{19,20}$. El PET/CT es la imagen de elección al evaluar la presencia de una masa, área de estenosis y el compromiso ganglionar ${ }^{21}$. La colonoscopía puede mostrar un compromiso variable de la mucosa, ya sea una masa, una ulceración o infiltración ${ }^{19}$ y permite obtener biopsias para un diagnóstico patológico.

El riesgo de linfoma se ha convertido en una variable importante a considerar al momento de definir las opciones terapéuticas en pacientes con EII. La inflamación crónica persistente puede jugar un rol en la promoción de células precursoras del linfoma, lo que llevaría a una sobreestimulación y una continua proliferación del sistema inmune ${ }^{22}$. Sin embargo, a diferencia de lo que sucede con la artritis reumatoidea ${ }^{14}$, la incidencia de LGIP en pacientes con EII es relativamente baja, indicando que la EII por sí sola no sería un factor de riesgo importante en el desarrollo del linfoma ${ }^{23}$. Otro factor de riesgo de linfoma es la infección por VEB, Dayshard y cols. mostraron que aquellos pacientes con EII tratados con tiopurínicos que posteriormente desarrollaron un linfoma presentaban con mayor frecuencia el VEB en las células tumorales ${ }^{24}$, lo cual pudimos demostrar en nuestro paciente. Por último, el uso de tiopurínicos y anti-TNF también se ha asociado a riesgo de linfoma, aunque la evidencia es escasa. En el caso de los tiopurínicos, estudios han sido contradictorios, probablemente por sesgo en la población estudiada y la duración de la terapia ${ }^{9,10,25,26}$. Estudios han sugerido un umbral de 4 años de duración de la terapia con tiopurínicos como un factor de riesgo para desarrollar un linfoma ${ }^{27,28}$, situación que no ocurrió en nuestro paciente. El riesgo de linfoma asociado al uso de anti-TNF en pacientes con EII es difícil de cuantificar, ya que la mayoría de los pacientes tiene tratamiento asociado con tiopurínicos o metotrexato ${ }^{29}$. Nuestro paciente sólo alcanzó a recibir dos dosis de infliximab, lo que hace altamente improbable su asociación con el linfoma rectal. Recientemente, un estudio poblacional mostró que ni el uso de tiopurínicos ni la terapia biológica se asociaron con el riesgo de desarrollar un linfoma ${ }^{30}$.

En relación al manejo del linfoma colorrectal primario, este requiere de una evaluación multidisciplinaria, donde el pronóstico y sobrevida del paciente está determinado por el estadio del linfoma. En relación a las alternativas de tratamiento, no existe un consenso sobre la mejor estrategia terapéutica, siendo las opciones cirugía, quimioterapia y radioterapia, ya sea como tratamiento único o combinado ${ }^{13,31-33}$. En nuestro paciente se eligió quimioterapia con esquema $\mathrm{R}-\mathrm{CHOP}$, alcanzando criterios de remisión completa tras 6 ciclos, sin complicaciones. En relación al efecto de la quimioterapia en la evolución de la EII, distintos estudios han demostrado su efecto beneficioso ${ }^{34,35}$. La evolución de nuestro paciente ha sido favorable, siendo evaluado tres meses después del término de su quimioterapia, encontrándose actualmente en remisión clínica (índice de Mayo clínico 0) y endoscópica (índice de Mayo endoscópico 1 a nivel de recto y sigmoides. Figura 2 d-f).

En conclusión, aunque el LGIP es infrecuente, este debe ser considerado en pacientes con EII que no presenten una evolución favorable. El rol de la EII y el tratamiento con tiopurínicos y anti-TNF en el desarrollo del linfoma es controversial.

\section{Referencias}

1. Freeman C, Berg JW, Cutler SJ. Occurrence and prognosis of extranodal lymphomas. Cancer 1972; 29: 252-60.

2. Koch P, del Valle F, Berdel WE, Willich NA, Reers B, Hiddemann W, et al. Primary gastrointestinal non-Hodking's lymphoma: I. Anatomic and histologic distribution, clinical features, and survival data of 371 patients registered in the German Multicenter Study GITHNL 01/92. J Clin Oncol 2001; 19: 3861-73.

3. Gurney KA, Gurney KA, Cartwright RA. Increasing incidence and descriptive epidemiology of extranodal non-Hodgkin lymphoma in parts of England and Wales. Hematol J 2002; 3: 95-104.

4. Ghimire P, Wu GY, Zhu L. Primary gastrointestinal lymphoma. World J Gastroenterol 2011; 17: 697-707.

5. Arora N, Manipadam MT, Pulimood A, et al. Gastrointestinal lymphomas: pattern of distribution and histological subtypes: 10 years experience in a tertiary centre in South India. Indian J Pathol Microbiol 2011; 54: 712-9. 
6. Ge Z, Liu Z, Hu X. Anatomic distribution, clinical features, and survival data of 87 cases primary gastrointestinal lymphoma. World J Surg Oncol 2016 Mar 18.

7. Wong MT, Eu KW. Primary colorectal lymphomas. Colorectal Dis 2006;8: 586-91.

8. Zighelboim J, Larson MV. Primary colonic lymphoma. Clinical presentation, histopathologic features, and outcome with combination chemotherapy. J Clin Gastroenterol 1994; 18: 291-7.

9. Beaugerie L, Brousse N, Bouvier AM, Colombel JF, Lémann M, Cosnes J, et al. Lymphoproliferative disorders in patients receiving thiopurines for inflammatory bowel disease: a prospective observational cohort study. Lancet 2009; 374: 1617-25.

10. Kandiel A, Fraser AG, Korelitz B, Bresinger C, Lewis JD. Increased risk of lymphoma among inflammatory bowel disease patients treated with azathioprine and 6-mercaptopurine. Gut 2005; 54: 1121-5.

11. Siegel CA, Marden SM, Persing SM, et al. Risk of lymphoma associated with combination anti-tumor necrosis factor and immunomodulator therapy for the treatment of Crohn's disease: a meta-analysis. Clin. Gastroenterol. Hepatol 2009; 7: 874-81.

12. Cai S, Cannizzo F Jr, Bullard Dunn KM, Gibbs JF, Czuczman M, Raiput A. The role of surgical intervention in non-Hodgkin's lymphoma of the colon and rectum. Am J Surg 2007; 193: 409-12.

13. Tevlin R, Larkin JO, Hyland JM, O'Connell PR, Winter DC. Primary colorectal lymphoma - a single centre experience. Surgeon 2015; 13: 151-5.

14. Baecklund E, Iliadou A, Askling J, Ekbom A, Backlin C, Granath F, et al. Association of chronic inflammation, not its treatment, with increased lymphoma risk in rheumatoid arthritis. Arth Reum 2006; 54: 692-701.

15. Smedby KE, Baecklund E, Askling J. Malignant lymphomas in autoimmunity and inflammation: a review of risks, risk factors, and lymphoma characteristics. Cancer Epidemiol Biomarkers Prev 2006; 15: 2069-77.

16. Dood GD. Lymphoam of the hollow abdominal viscera. Radiol Clin North Am 1990; 28: 771-83.

17. Quayle FJ, Lowney JK. Colorectal lymphoma. Clin Colon rectal surg 2006; 19: 49-53.

18. Shepherd NA, Hall PA, Coates PJ, Levison DA. Primary malignant lymphoma of the colon and rectum. A histopathological and immunohistochemical analysis of 45 cases with clinicopathological correlations. Histopathology 1988; 12: 235-52.

19. Wang MH, Wong JM, Lien HC, Lin CW, Wang CY. Colonoscopic manifestations of primary colorectal lymphoma. Endoscopy 2001; 3: 605-9.

20. Kim YH, Lee JH, Yang SK, Kim TI, Kim JS, Kim HJ, et al. Primary colon lymphoma in Korea: a KASID (Korean association for the study of intestinal disease) study. Dig Dis Sci 2005; 50: 2243-7.

21. Lee HJ, Han JK, Kim TK, Kim YH, Kim AY, Kim KW, et al. Primary colorectal lymphoma: spectrum of imaging findings with pathologic correlation. Eur Radiol 2002; 12: 2242-9.

22. Copie-Bergman C, Niedobitek G, Mangham DC, Selves J, Baloch K, Diss TC, et al. Epstein-Barr virus in B-cell lymphomas associated with chronic suppurative inflammation. J Pathol 1997; 183: 287-92.

23. Watanabe N, Sugimoto N, Matsushita A, Maeda A, Nagai K, Hanioka K, et al. Association of intestinal malignant lymphoma and ulcerative colitis. Intern. Med. 2003; 42: 1183-7.

24. Dayharsh GA, Loftus EV Jr, Sandborn WJ, Tremaine WJ, Zinsmeister AR, Witzig TE, et al. Epstein-Barr virus-positive lymphoma in patients with inflammatory bowel disease treated with azathioprine or 6-mercaptopurine. Gastroenterology 2002; 122: 72-7.

25. Armstrong RG, West J, Card TR. Risk of cancer in inflammatory bowel disease treated with azathioprine: a UK population-based case-control study. Am J Gastroenterol 2010; 105: 1604-9.

26. Lakatos PL, Lovasz BD, David G, Pandur T, Erdelyi Z, Master O, et al. The risk of lymphoma in patients with inflammatory bowel diseases: results from a population-based cohort in Eastern Europe. J Crohn Colitis 2013; 7: 385-91.

27. Khan N, Abbas AM, Lichtenstein GR, Loftus Jr EV, Bazzano LA. Risk of lymphoma in patients with ulcerative colitis treated with thiopurines: a nationwide retrospective cohort study. Gastroenterology 2013; 145: 1007-15.

28. Beigel F, Steinborn A, Schnitzler F, Tillack C, Breiteneicher S, John JM, et al. Risk of malignancies in patients with inflammatory bowel disease treated with thiopurines or anti-TNF alpha antibodies. Pharmacoepidemiol Drug Saf 2014; 23: 735-44.

29. Lichtenstein GR, Feagan BG, Chen RD, Salzberg BA, Diamond RH, Langhoff W, et al. Drug therapies and the risk of malignancy in Crohn's disease: Results from the TREAT ${ }^{\mathrm{TM}}$ registry. Am J Gastroenterol 2014; 109: 212-23.

30. Chaparro M, Ramas M, Benítez JM, López-García A, Juan A, Guardiola J, et al. Extracolonic cancer in inflammatory Bowel Disease: Data from the GETTECCU Eneida Registry. Am J Gastroenterology 2017; 112: 1135-43.

31. Tang TC, Kuo MC, Chang H, Dunn P, Wang PN, Wu $\mathrm{JH}$, et al. Primary colonic lymphoma: an analysis of 74 
cases with localized large-cell lymphoma. Eur J Haematol 2011; 87: 28-36.

32. Kim SJ, Kang HJ, Kim JS, Oh SY, Choi CW, Lee SI, et al. Comparison of treatment strategies for patients with intestinal diffuse large B-cell lymphoma: surgical resection followed by chemotherapy versus chemotherapy alone. Blood 2011; 117: 1958-65.

33. Jeong JH, Kim S, Yoon DH, Lee SW, Huh J, Suh C. Clinical characteristics, treatment, and outcome of pri- mary rectal lymphoma: a single center experience of 16 patients. Blood Res 2017; 52: 125-9.

34. Barta Z, Tóth L, Zeher M. Pulse cyclosphosphamide therapy for Inflammatory Bowel Disease. World J Gastroenterol 2006; 12: 1278-80.

35. Axelrad JE, Fowler SA, Friedman S, Ananthakrishnan A, Yajnik V. Effects of cancer treatment on Inflammatory Bowel Disease. Clin Gastroenterol Hepatol 2012; 10: 1021-7. 